\title{
Disturbance Rejection Controller Design Based on Sensitivity Function Notching
}

\author{
Hai Ho \\ Kennesaw State University
}

\begin{abstract}
This paper presents a feed-forward control scheme for rejecting stochastic narrow band disturbances. The effect of this scheme manifests itself as a tunable notch on the sensitivity transfer function frequency response, where the target frequency, width, and depth of the notch can be set by prescribed design parameters. This approach is based on employing least mean square adaptive control that subsequently resulted in a linear time invariant (LTI) controller. The appeal of this approach includes the ability to directly notch the sensitivity function and ease of implementation on digital control platforms. This scheme was used in actual high volume hard disk drive (HDD) products.
\end{abstract}

Keywords: HDD, Control System, Servo, Disturbance Rejection

\section{Introduction}

There are many control applications where the disturbance or command signals are in the form of stochastic resonance. Take for example, hard disk drive with motor bearing and disk flutter resonance modes that contain very high concentrated energy that contribute significantly to large position tracking error $[4,5]$. In practice, sometimes a control engineer would attempt to place a generic peaking filter in the primary feedback or feed forward path to give extra gain at the offending frequency range. But this doesn't always work due to the sensitivity of the phase and gain on the sensitivity error function, therefore, resulting in lob sided waterbed effect. Furthermore, the task of rejecting disturbance near the cross-over frequencies has been even more difficult.

This paper presents a practical method to deal with stochastic narrow band disturbances. The end result is achieving a notch characteristic locally on the sensitivity error transfer function. The approach is based on modification of prior work on adaptive feed-forward control (AFC) [1].

This technique was used in an actual disk drive product that was manufactured in high volume of over fifty million units a year. The system successfully suppressed the resonance ringing during settle mode of the actuator seeking to a data track [2].

\section{Problem Statement and Formulation}

\subsection{Problem Statement}

Consider the block diagram of a general digital control system with the controller, plant, and disturbances labelled in Fig. 1. The controller, $\mathrm{C}(\mathrm{z})$, is predesigned to achieve certain target metrics such as bandwidth, phase margin, and gain margin. However, consider there are narrow band disturbances $d_{i}(k)$ and/or $d_{o}(k)$ present. In this paper, we propose a way to directly and substantially rejects such disturbances.

The rejection of disturbances are governed by the following respective output and input sensitivity transfer functions:

$$
\begin{aligned}
& \frac{E(z)}{D_{o}(z)}=S(z) \\
& \frac{E(z)}{D_{i}(z)}=P(z) S(z)
\end{aligned}
$$


Where

$$
S(z)=\frac{1}{1+C(z) P(z)}
$$

An example of the output sensitivity function frequency response is shown in Fig. 2. If the disturbance is at frequencies below the $0 \mathrm{~dB}$ crossover $\mathrm{f}_{1}$, it get rejected by the system. At frequencies between $\mathrm{f}_{1}$ and $\mathrm{f}_{2}$, disturbances actually get amplified because the error gain is higher $0 \mathrm{~dB}$. Lastly frequencies above $\mathrm{f}_{2}$, disturbances get no rejection at all.

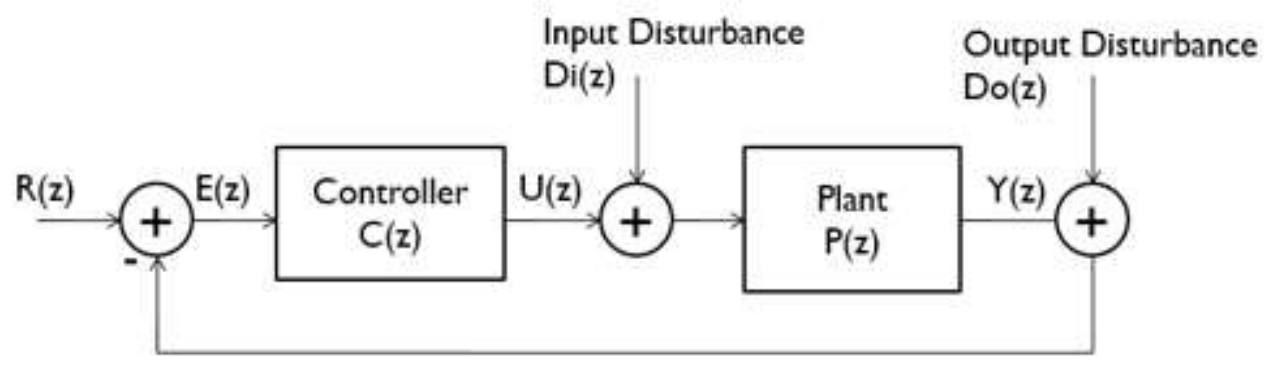

Fig. 1: Closed loop control system diagram.

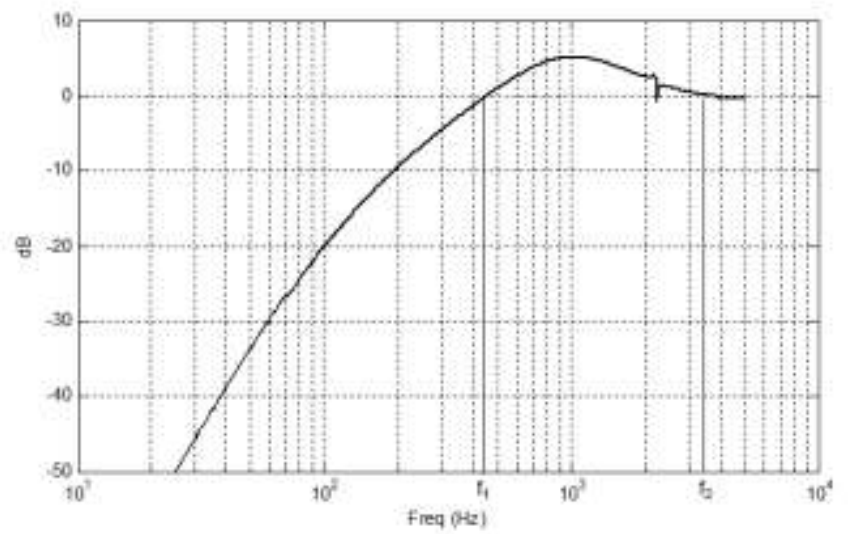

Fig. 2: Output error sensitivity transfer function bode plot.

We examine the case of output disturbance that is narrow band and can be described as

$$
d_{i}(k)=A(k) \cos \left(\omega_{o} k T+\theta(k)\right)
$$

where $A(k)$ and $\theta(k)$ are stochastic unknown parameters, and $\omega_{o}$ is the nominal resonant frequency that is known a priori.

\subsection{Formulation}

The nominal control system in Fig. 1 can be enhanced by adding an adaptive feed-forward compensator as shown in Fig. 3 [1]. The idea here is to generate a feed-forward control signal $u_{f f}(k)$ from sinusoidals at the known frequency with gains, $[a(k) b(k)]^{\prime}$ adapted by LMS algorithm that minimizes the error signal in the presence of the narrow band output disturbance. 


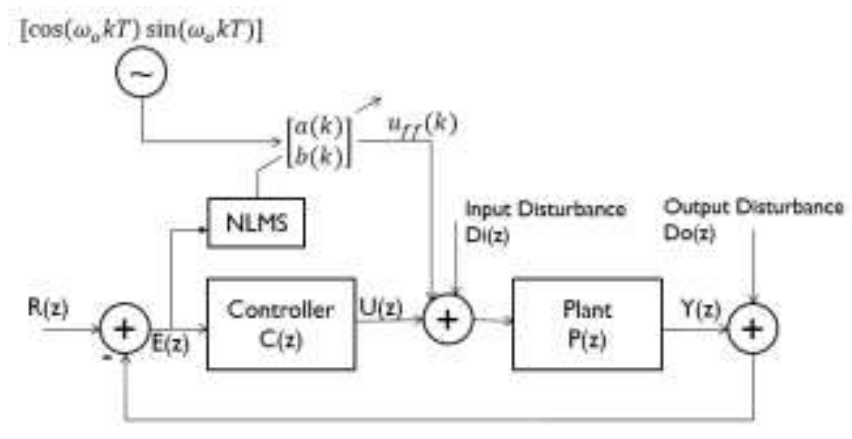

Fig. 3: Adaptive feed forward architecture.

The feed forward control law can be expressed as

$$
u_{f f}(k)=a(k) \sin \left(\omega_{o} k T\right)+b(k) \cos \left(\omega_{o} k T\right)
$$

In minimizing the cost function, $J=E\left\{e^{2}(t)\right\}$, where e(t) is the time domain feedback error signal, the feed forward gains can be adapted as

$$
\begin{aligned}
& a(k+1)=a(k)+\mu \alpha \cos \left(\omega_{o} k T+\varphi\right) \\
& \quad b(k+1)=b(k)+\mu \alpha \sin \left(\omega_{o} k T+\varphi\right),
\end{aligned}
$$

Where $\mu$ is the learning rate, and with the nominal closed loop system using $\mathrm{C}(\mathrm{z})$. It can be shown that the two loop matching parameters are

$$
\begin{aligned}
& \alpha=\left|P\left(e^{j \omega T}\right) S\left(e^{j \omega T}\right)\right|_{\omega=\omega_{o}} \\
& \varphi=\arg \left|P\left(e^{j \omega T}\right) S\left(e^{j \omega T}\right)\right|_{\omega=\omega_{o}} .
\end{aligned}
$$

These parameters are usually determined a priori. Particularly, $\varphi$ is crucial in achieving a fairly symmetrical notch on the sensitivity frequency response, as we will show. It was shown that this type of control law can has an equivalent LTI representation [1]. For (4)-(5), the LTI equivalent is

$$
\frac{U_{f f}(z)}{E(z)}=\mu \alpha \frac{z^{2}[\cos (\varphi)]-z\left[\cos \left(\varphi+\omega_{o} T\right)\right]}{z^{2}-z\left[2 \cos \left(\omega_{o} T\right)\right]+1}
$$

A first observation is that this is a peaking filter with the phase matched to the nominal control loop via $\varphi$. The poles of this filter is right on the unit circle, therefore, infinite gain and very narrow, which is not too useful. If we introduce a scaling factor at the denominator to get $z^{2} / \gamma-z\left[2 \cos \left(\omega_{0} T\right)\right]+\gamma$, we can move the poles slightly inside the unit circle to reduce the peak while broadening the width. This shall become a useful tuning parameter. A second observation is that we could normalize the gain of this filter such that it is benign at frequencies outside of the target area, $\omega_{0}$. This is equivalently setting the gain parameter to achieve $0 \mathrm{~dB}$ dc gain, which can be done as

$$
\frac{U_{f f}(z)}{E(z)}=K \frac{z^{2}[\cos (\varphi)]-z\left[\cos \left(\varphi+\omega_{o} T\right)\right]}{\frac{z^{2}}{\gamma}-z\left[2 \cos \left(\omega_{o} T\right)\right]+\gamma}=A(z)
$$

with the gain 


$$
K=\left|\frac{z^{2}[\cos (\varphi)]-z\left[\cos \left(\varphi+\omega_{o} T\right)\right]}{\frac{z^{2}}{\gamma}-z\left[2 \cos \left(\omega_{o} T\right)\right]+\gamma}\right|_{z=0}
$$

This feed-forward control architecture is shown in Fig. 4.

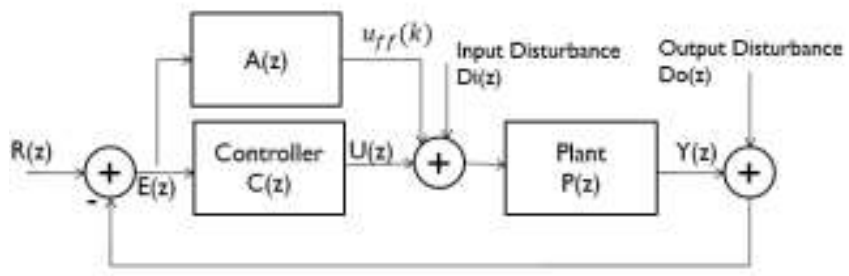

Fig. 4: Equivalent feed-forward architecture.

The controller $\mathrm{A}(\mathrm{z})$ is a second order digital filter that is fairly simple to implement. Furthermore, this configuration is convenient for applications where $\mathrm{A}(\mathrm{z})$ can be switched on or off without adverse disruption to the nominal closed loop. An example of this was in HDD settle control [2], where A(z) was activated only during settle mode, and then turned off during track follow mode. The corresponding sensitivity function of Fig. 4 is

$$
S_{2}(z)=\frac{1}{1+[A(z)+C(z)] P(z)}
$$

We next simulate this scheme on a HDD control problem. A HDD is a suitable application since it has numerous narrow band disturbances such disk flutter and motor bearing resonances [4,5]. A system with $\mathrm{P}(\mathrm{z})$ and $\mathrm{C}(\mathrm{z})$ are given in [5]. The actuator has resonances around $2.2 \mathrm{Khz}$, and the nominal controller achieves open loop bandwidth of $630 \mathrm{~Hz}$. The digital control sampling rate is $14.3 \mathrm{Khz}$. For the feedfoward filter, we set $\omega_{o}$ to be 1.0 KHz. Figure 6 shows the comparison of the sensitivity function with and without $A(z)$, as described in (2) and (9), respectively.

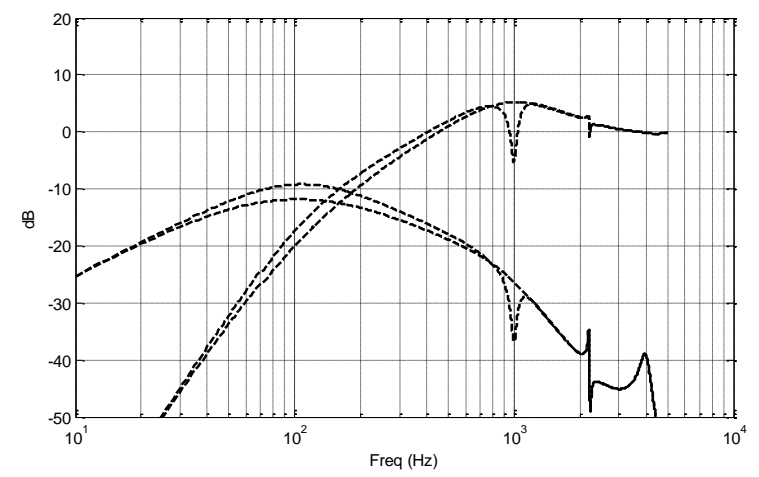

Fig. 5: Comparing sensitivity transfer functions (1a,b) with (solid) and without (dashed) feedfoward A(z).

Here we see that a localized notch profile was produced on the sensitivity function at $\omega_{o}$, which indicates a deeper rejection of disturbances at the target frequency. Notice that the notch is fairly symmetrical and does not cause serious lopsided waterbed effect. This is due to the use of the loop shaping parameter $\varphi$ in (8). The waterbed effect is clearly present, showing that the area under the curves does not change, where "dirt" was moved from one area to the other, as described in [3]. This is indeed a tradeoff in which rejection in neighboring lower frequencies are slightly reduced. The depth and width of the notch can be affected by the parameter $\gamma$, 
which should be between .97 to 1 . The closer it is to 1 , the narrower and deeper the notch becomes. In this example on Fig. 5, $\gamma$ was picked to be 0.99 .

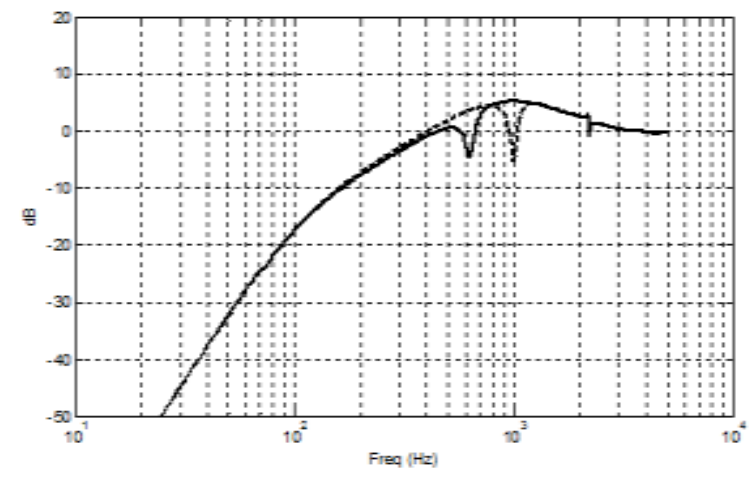

Fig. 6: Notch placed at gain cross-over frequency $630 \mathrm{~Hz}$ (solid), and $1 \mathrm{Khz}$ (dashed).

We next demonstrate the robustness of deploying $\mathrm{A}(\mathrm{z})$ at a gain cross over frequency, $630 \mathrm{~Hz}$. The simulation in Fig. 6 shows that a sensitivity notch (solid) was indeed placed at this frequency without serious impact the stability margins and shape of this function outside of the target frequency, $\omega_{0}$.

\section{Design and Application}

The control system architecture in Fig. 4 is practical for implementation on embedded or digital control platforms. The feed forward controller, $\mathrm{A}(\mathrm{z})$, is suitable for stochastic disturbances that are narrow band, such as resonance modes. This controller is effective for rejecting both output and input disturbances, as well as tracking narrow band reference signal. A control system engineer can follow these design steps.

\section{Design Procedure:}

1. Design nominal controller, $C(z)$

2. Identify the frequency of the disturbance, $\omega_{0}$

3. Determine (measure or simulate) the phase, $\varphi$ in $(6 b)$

4. Synthesize $A(z)$ in (8) with tuning the notch width and depth via $\gamma$.

5. Simulate or implement complete control system

This approach was successfully used in HDD products [2] where the spindle motor exhibited resonances that resulted in oscillation during seek settling. After employing this feed forward filter, the greater than $80 \%$ oscillation was eliminated. This enabled products to be shipped in high volume of over fifty million units a year. In the full paper version, we will show more detailed results.

\section{Summary}

In this paper, it was shown how a notch can be placed on the sensitivity error frequency response to achieve deeper rejection of disturbances that are stochastic and narrow band, such as resonances. The ability to effectively place a notch on the sensitivity at an identified offending target frequency is a very useful control technique. Furthermore, the scheme is simple and in a form of of an LTI second order feedforward filter that can be readily implemented in digital control systems. The mathematical formulation was based on extending the AFC framework [1] and [2]. Here, we introduce the tuning parameters and examine the effect on the sensitivity frequency response. This control scheme can be generalized to include multiple modes by replicating the matched peaking filter at the additional frequencies. This scheme is turn-key such that automation and calibration can be done fairly readily. 


\section{References}

[1] Bodson, M., Sacks, A., \& Khosla, P. (1994). Harmonic generation in adaptive feedforward cancellation schemes. Automatic Control, IEEE Transactions on,39(9), 1939-1944. http://dx.doi.org/10.1109/9.317130

[2] Ho, H. T., Won, J., Abou-Jaoude, F. Y., \& Campbell, G. A. (2005). U.S. Patent No. 6,937,428. Washington, DC: U.S. Patent and Trademark Office.

[3] Doyle, J. C., Francis, B. A., \& Tannenbaum, A. (1992). Feedback control theory (Vol. 1). New York: Macmillan Publishing Company.

[4] Young, P. M., Morris, J. C., \& Ho, H. T. (2003, June). Servo control of a dual-stage actuator for a high performance disk drive. Part 1: modeling and simulation. In American Control Conference, 2003. Proceedings of the 2003(Vol. 3, pp. 2523-2528). IEEE.

[5] Franklin, G. F., Workman, M. L., \& Powell, D. (1997). Digital control of dynamic systems. Addison-Wesley Longman Publishing Co., Inc. 